\title{
Synthesis and Structures of Bulk Nanocrystalline Copper
}

\author{
K. Zhang *, J. R. Weertman * and P. L. Gai **,*** \\ * Department of Materials Science and Engineering, Northwestern University, Evanston, IL \\ 60208 \\ ** Central Research and Development Laboratories, DuPont, Wilmington, DE 19880-0356; and \\ *** Department of Materials Science and Engineering, University of Delaware, Newark DE.
}

Nanocrystalline metals are important because they exhibit exceptionally high strengths and therefore have generated considerable interest [1,2]. Nanocrystalline copper samples were made by inert gas condensation (IGC) and in situ compaction in equipment at Argonne National Laboratory. They were made from $99.999 \%$ pure $\mathrm{Cu}$ stock. The average grain size of each sample was determined from transmission electron microscopy observations of $\sim 500$ grains. The grain size distribution was quite narrow. The samples had a density of $98-99 \%$ of the coarse-grain value [1]. Vacuum conditions and helium purity have recently been improved further by an order of magnitude or greater. Sample hardness was measured with a microhardness tester, as a function of dwell time of the hardness indenter in the sample over dwell times from $5 \mathrm{~s}$ to several hours and temperatures ranging from room temperature (RT) liquid nitrogen temperature (LN2) (figure 1).

We have obtained structural and chemical data of grains and grain boundaries at the atomic resolution and on the nanoscale, respectively, using FEI FE(S)TEM Tecnai and CM30 Environmental-TEM (ETEM)[3] instruments. Grain boundary compositions were determined using the electron nanoprobe in the STEM mode. The data are quite informative. Our studies of the RT sample have shown grain sizes of $\sim 15-70 \mathrm{~nm}$ in areas away from the indented regions and 80nm-180 nm near the indented regions, suggesting grain growth near the indents. High concentrations of dislocations were observed (figure 2). The high internal strains seen in figure 2 come from the built-in strains in the nanoclusters before compaction caused by cluster coalescence and/or from the deformation produced by the indenter. In the LN2 sample, we have observed much larger grains varying from 0.1 microns to about 0.5 microns. We have found fewer grain boundaries and fewer dislocations than in the RT sample. Coherent boundaries between two (110) grains and atomically flat boundaries between (110), (111) and (010) grains were observed in both the samples. Atomic resolution imaging has revealed clean grain boundaries (figure 3, between (111) grains showing 110 lattice planes). Grains exhibit Moire fringes due to overlapping grains. Atomic scale twinning along $<111>$ in (110) grains were observed (shown in figure 4 in LN2 sample) and along $<110>$ in (111). The atomic structure and nano-probe analyses using $\mathrm{X}$-ray spectroscopy (EDX) are consistent with $\mathrm{Cu}$, with no impurity segregation at the grain boundaries (figure 5).

\section{References:}

[1] P. G. Sanders, J. A. Eastman and J.R. Weertman, Acta Mater. 45 (1997) 4019.

[2] P.L. Gai, R. Mitra and J.R. Weertman, Pure and Appl. Chem. (IUPAC) 74 (2002)1519.

[3] P.L. Gai and E D Boyes, Electron Microscopy in Het. Catalysis, IOPP, UK, USA, 2003. 


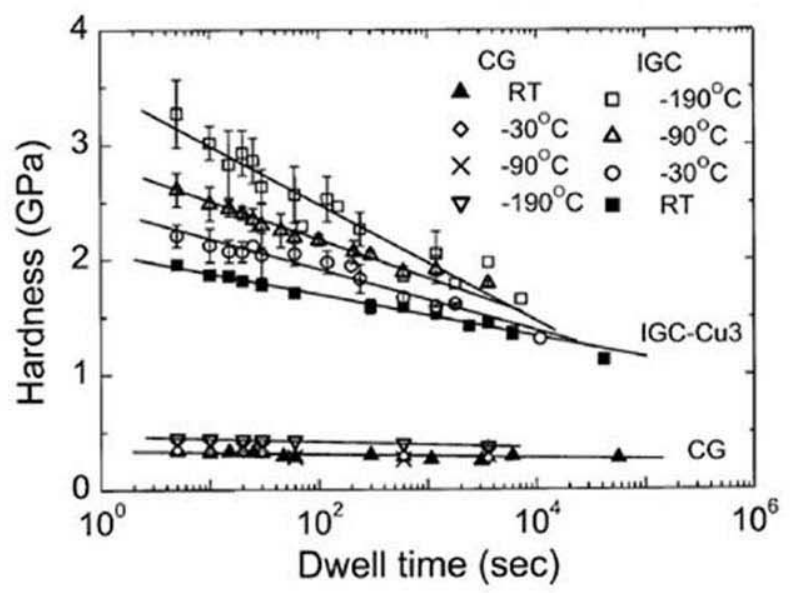

Fig.1
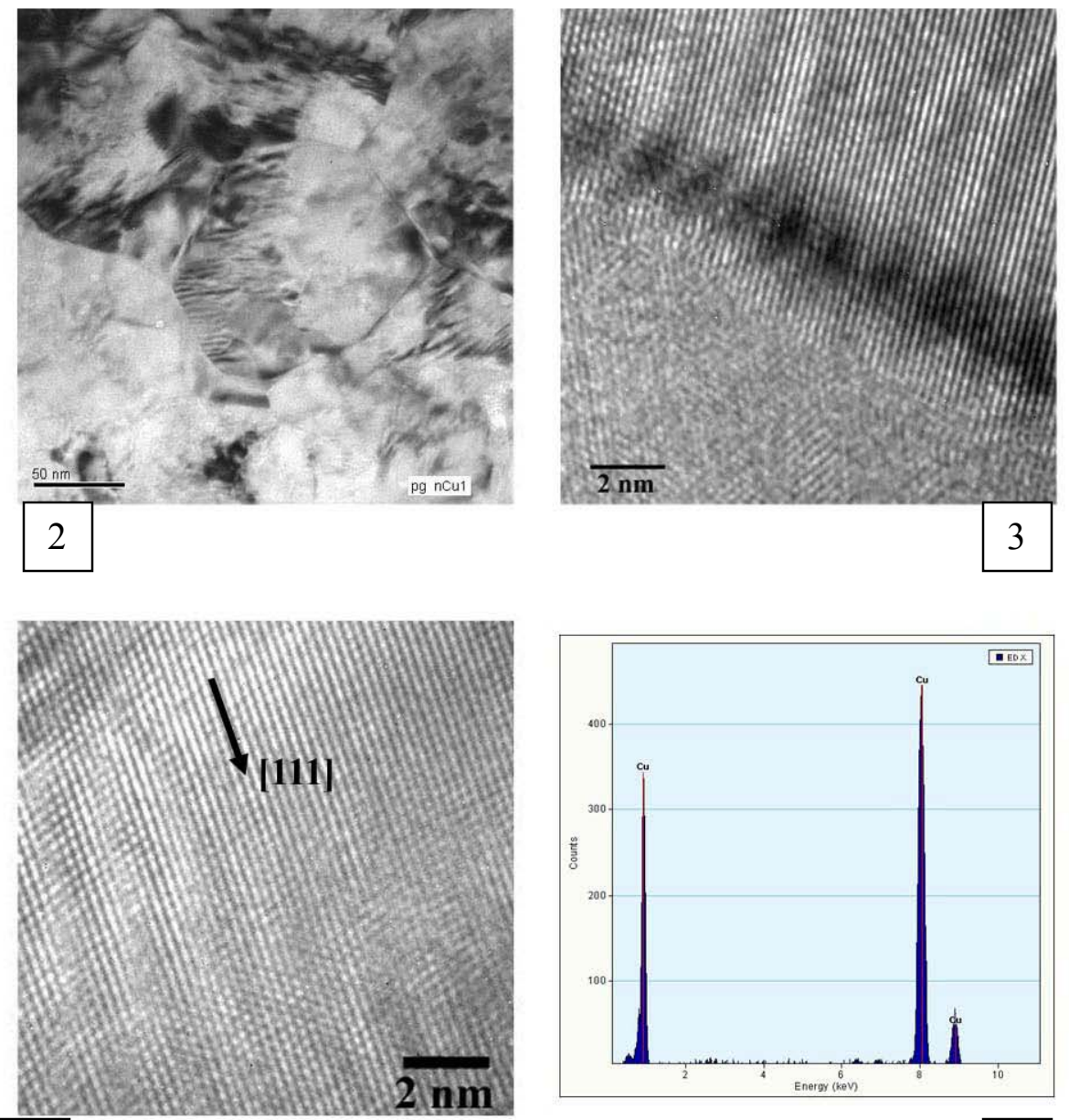

4

Fig.1 Measurements of hardness of nanocrystalline copper as a function of dwell time of the hardness indenter in the sample over dwell times and temperatures Fig.2. High concentrations of dislocations near indented regions

Fig.3. Atomic resolution image of grain boundaries between (111) grains

Fig.4. Nanoscale twinning along $<111>$ in (110) grains

Fig.5. STEM nano-probe analyses show clean grain boundaries with no impurities 